\section{Adriano Mondini'}

Francisco Chiaravalloti Neto"

\section{Variáveis socioeconômicas e a transmissão de dengue}

\section{Socioeconomic variables and dengue transmission}

\section{RESUMO}

OBJETIVO: Avaliar a relação entre o risco de ocorrência de dengue e os níveis socioeconômicos.

MÉTODOS: Os casos autóctones de dengue confirmados entre setembro de 1990 a agosto de 2002 foram geocodificados e agrupados segundo setores censitários urbanos de São José do Rio Preto, estado de São Paulo. Um fator socioeconômico foi gerado por meio da técnica de análise de componentes principais, agrupando os setores censitários em quatro níveis socioeconômicos. Os coeficientes de incidência foram calculados, por ano e quadriênio, para cada um dos agrupamentos de setores censitários, considerando-se o período entre setembro de um ano a agosto do ano seguinte. São apresentados mapas temáticos dos setores agrupados nos quatro níveis socioeconômicos com os respectivos coeficientes de incidência da doença.

RESULTADOS: A análise de componentes principais produziu um fator socioeconômico responsável por $87 \%$ da variação total. Esse fator esteve associado com as incidências de dengue apenas no ano de 1994-1995.

CONCLUSÕES: A ausência de associação encontrada entre risco de ocorrência de dengue e níveis socioeconômicos na quase totalidade dos anos estudados mostra que esta é uma questão que precisa ser mais bem estudada e, talvez, dependa da realidade de cada município. É importante que sejam verificadas as relações espaciais entre a transmissão de dengue e outras variáveis, como o grau de imunidade da população; a efetividade das medidas de controle; o grau de infestação pelo vetor; os hábitos e atitudes da população, entre outros.

DESCRITORES: Dengue, epidemiologia. Fatores de risco. Fatores socioeconômicos. Sistemas de informação geográfica.
Faculdade de Medicina de São José do Rio Preto. São José do Rio Preto, SP, Brasil

" Superintendência de Controle de Endemias. São José do Rio Preto, SP, Brasil

Correspondência | Correspondence: Francisco Chiaravalloti Neto

Av. Brigadeiro Faria Lima, 5416

15090-000 São José do Rio Preto, SP, Brasil

E-mail: fcneto@famerp.br 


\section{ABSTRACT}

OBJECTIVE: To evaluate the relationship between risk of occurrence of dengue and socioeconomic level.

METHODS: All confirmed autochthonous cases of dengue between September 1990 and August 2002 were geocoded and grouped according to the urban census tracts of the municipality of São José do Rio Preto, Southeastern Brazil. A socioeconomic factor generated by principal component analysis was used to group census tracts into four socioeconomic levels. Incidence rates were calculated for each year and four-year period for each of the census sectors, considering the period from September of one year to August of the next. Thematic maps of sectors, grouped into each of the four socioeconomic levels and their respective disease incidences, are presented.

RESULTS: Principal component analysis generated a socioeconomic factor that accounted for $87 \%$ of total variation. This factor was associated with dengue incidence only for the 1994-95 period.

CONCLUSIONS: The lack of an association between risk of occurrence of dengue and socioeconomic levels in almost all years studied indicates that this issue deserves further study, and may vary depending on the settings found in each municipality. It will be important to determine the spatial relationship between dengue transmission and other variables, such as degree of immunity in the population, effectiveness of control measures, degree of infestation by the vector; and population habits and behaviors, among others.

Key words: Dengue, epidemiology. Risk factors. Socioeconomic factors. Geographic information systems.

\section{INTRODUÇÃO}

A dengue, cuja manifestação mais grave é a dengue hemorrágica, é considerada uma doença infecciosa em intensa expansão no mundo. ${ }^{16}$ Cerca de três bilhões de pessoas encontram-se em risco de contrair o vírus e anualmente são registrados 50-100 milhões de casos de dengue clássica e cerca de 500 mil internações por febre hemorrágica, chegando a uma mortalidade de $5 \%$ nestes casos. ${ }^{*}$ A dengue configura-se como um problema de saúde pública, gerando gastos anuais de milhões de dólares. ${ }^{16}$

$O$ vírus da dengue é numericamente o mais importante arbovírus humano; possui quatro sorotipos diferentes (DENV 1, 2, 3 e 4) transmitidos no ambiente urbano pelo mosquito Aedes aegypti. ${ }^{18} \mathrm{O}$ vetor está amplamente associado às atividades antrópicas, que disponibilizam sítios de oviposição artificiais e permitem a manutenção de sua infestação. Os centros urbanos configuram-se como favorecedores da dispersão e aumento da densi- dade do mosquito, já que o espaço social organizado influencia a interação entre o vetor, vírus e homem. ${ }^{20}$

Diversos fatores de risco estão relacionados com presença da doença e do vetor. Tauil ${ }^{21}$ destaca como fatores fundamentais para definir o padrão de transmissão: crescimento populacional, migrações, viagens aéreas, urbanização inadequada, mau funcionamento dos sistemas de saúde e densidade populacional.

O Brasil foi responsável por $56 \%$ dos casos de dengue notificados nas Américas entre 2001 e 2005.** Foram registrados 241.796 casos de dengue clássica e 43 óbitos pela forma hemorrágica da doença em 2005. Em relação ao ano anterior, houve aumento de aproximadamente 95\% na média nacional.*** O País apresentou um comportamento cíclico: em alguns anos incidências mais altas e outros com incidências mais baixas, tendência também apresentada pelo Estado de São Paulo. Em 2004 e 2005 foram registrados, respectivamente,

* World Health Organization. Dengue factsheet, 2005. Geneva; 2005. [6/6/2006] Disponível em: http://www.who.int/mediacentre/ factsheets/fs117/en/

** Pan American Health Organization. 2005: Number of reported cases of dengue \& dengue hemorraghic fever (DHF), region of Americas (by country and subregion). [6/6/2006] Disponível em: http://www.paho.org/English/AD/DPC/CD/dengue-cases-2005.htm

*** Ministério da Saúde. Secretaria de Vigilância em Saúde. Brasília; 2007: Gráficos de evolução das doenças (1980 - 2005). [29/10/2007]

Disponível em: http://portal.saude.gov.br/portal/saude/visualizar_texto.cfm?idtxt=25340 
3.049 e 5.433 casos de dengue e até abril de 2006, foram registrados 5.767 casos no Estado.*

Estudo** realizado na região noroeste do Estado de São Paulo, incluindo o município de São José do Rio Preto, confirmou essa tendência cíclica e a importância das cidades médias como locais com maior probabilidade de ocorrência da doença, exercendo o papel de irradiadores da transmissão. Mondini et $\mathrm{al}^{13}$ caracterizaram o processo de endemização da dengue no município de São José do Rio Preto, mostrando que a transmissão da doença não ocorre uniformemente em todo o município. Há necessidade de realização de investigações sobre o papel das populações humanas e suas condições socioeconômicas na manutenção da infestação do vetor e circulação viral. ${ }^{20}$ Dessa forma, o objetivo do presente trabalho foi avaliar a relação entre o risco de ocorrência de dengue e os níveis socioeconômicos nos setores censitários urbanos.

\section{MÉTODOS}

A cidade de São José do Rio Preto está entre as cidades brasileiras com qualidade de vida comparável a países desenvolvidos, localizada na região noroeste do estado de São Paulo, a $452 \mathrm{~km}$ da capital e a $489 \mathrm{~m}$ acima do nível do mar (2049' '1" latitude sul e 49²2'46" longitude oeste). A área total do município é de 434,10 $\mathrm{km}^{2}$, a área urbana ocupa $96,81 \mathrm{~km}^{2}$, com população de 406.826 habitantes estimada para o ano de 2006. O clima de São José do Rio Preto é tropical, com temperatura média de $25^{\circ} \mathrm{C}$, pluviosidade anual por volta de $1.410 \mathrm{~mm}$.

O mosquito transmissor da dengue foi novamente identificado no município em 1985 e, entre este ano e 1989, ocorreram apenas casos importados da doença. Os primeiros casos autóctones ocorreram em 1990, quando houve a introdução do vírus DEN 1. Os sorotipos DEN 2 e DEN 3 foram introduzidos em 1997 e 2003, respectivamente.***

O banco de dados incluiu os casos autóctones de dengue notificados à Secretaria Municipal de Saúde e Higiene de São José do Rio Preto entre setembro de 1994 a agosto de 2002, e confirmados laboratorialmente pelo Instituto Adolfo Lutz. Considerou-se a data de início dos sintomas como a data do caso.

A geocodificação dos casos autóctones de dengue foi realizada com auxílio das ferramentas do Programa ArcGis 9.0 (ESRI), com a utilização da Base
Cartográfica de São José do Rio Preto (em projeção Universal Transversa de Mercator - UTM) com eixos de ruas fornecidos pela Prefeitura Municipal. Depois de geocodificados, os casos foram agrupados segundo os 432 setores censitários da área urbana, segundo a Fundação Instituto Brasileiro de Geografia e Estatística (IBGE). **** Para a avaliação de transmissão da dengue optou-se pela utilização dos setores censitários como nível de agregação, por serem delimitados geograficamente e mostrarem características homogêneas.

As variáveis socioeconômicas utilizadas para a realização da análise de componentes principais foram selecionadas de acordo com estudo desenvolvido por Sposati***** e modificado por Vendramini et al. ${ }^{24}$ As variáveis foram fornecidas pelo $\mathrm{IBGE}^{* * * *}$ e estão relacionadas a: renda e anos de instrução médios das pessoas responsáveis pelos domicílios; renda e anos de instrução médios das mulheres responsáveis pelos domicílios; proporção de pessoas e de mulheres analfabetas; proporção de domicílios com cinco ou mais moradores. A escolha dos dados socioeconômicos relativos ao ano 2000 foi uma estratégia metodológica para minimizar os erros que pudessem advir de dados incompletos para a análise. Isso porque houve um aumento do número de setores censitários entre 1990-1994 e a utilização de dados anteriores a esse período não contemplaria as novas regiões que surgiram no município.

A análise das componentes principais com o programa Stata 7.0 produziu vários fatores, tendo sido escolhido o responsável pela maior proporção de variação total, denominado fator socioeconômico. Esse fator caracterizou os setores censitários, de forma que quanto maior seu valor, melhor o nível socioeconômico dos moradores do setor censitário. Seu valor para um determinado setor censitário foi calculado multiplicando-se cada um dos valores das variáveis pelos respectivos pesos fatoriais obtidos da análise de componentes principais e pela soma dos resultados obtidos. Os setores censitários foram agrupados em quartis, utilizando-se o fator gerado. Aqueles com o valor mais alto foram denominados nível 1 e os de valores mais baixos, nível 4, representando o melhor e o pior agrupamento socioeconômico, respectivamente. Os setores que apresentaram os valores intermediários foram denominados níveis 2 e 3 , representantes de estrato socioeconômico médio.

O período anual abrangeu setembro de um ano a agosto do ano seguinte. Essa divisão do ano possibilita uma boa representação da sazonalidade da doença, pois em geral os meses de setembro e agosto apresentam os

\footnotetext{
* Secretaria de Estado da Saúde de São Paulo. Centro de Vigilância Epidemiológica "Prof. Alexandre Vranjac", dados nao publicados.

** Pereira CA. Transmissão de dengue na região noroeste do estado de São Paulo [dissertação de mestrado]. São Paulo: Faculdade de Medicina de São José do Rio Preto; 2005.

*** Instituto Adolfo Lutz, dados não publicados.

**** Statcart. Version 1.1 [software em CD ROM]. Rio de Janeiro: Instituto Brasileiro de Geografia e Estatística; 2002.

***** Sposati A. Mapa da exclusão/inclusão social da cidade de Santo André. Santo André: Pontifícia Universidade Católica de São Paulo; 2000. [4/8/2005] Disponível em: http://www.dpi.inpe.br/geopro/exclusao/oficinas/mapa2000.pdf
} 
menores valores de incidência em relação aos demais meses do ano. O período entre os meses janeiro-abril apresenta as maiores incidências da doença, coincidindo com o aumento das precipitações pluviométricas.

Os coeficientes de incidência anuais foram calculados pela divisão do total de casos de cada período pela respectiva estimativa de população e multiplicação dos resultados por 100.000. Dessa forma, obteve-se uma série histórica de incidências anuais para cada um dos agrupamentos de setores censitários.

Os coeficientes de incidências quadrienais foram calculados dividindo-se o total de casos de cada conjunto de anos pela respectiva estimativa de população e multiplicando esses resultados por 100.000. Obteve-se uma série quadrienal de coeficientes de dengue para os períodos entre setembro a agosto de 1994 a 2002 para os quatro agrupamentos de setores censitários. Todas as estimativas anuais de população foram obtidas do Datasus.*

Mapas temáticos foram confeccionados com o programa ArcGis 9.0 (ESRI), a partir da malha de setores censitários, mostrando os quatro agrupamentos de setores e seus respectivos valores dos coeficientes de incidência da doença.

\section{RESULTADOS}

De um total de 14.431 casos autóctones de dengue ocorridos na área urbana, foram geocodificados 13.998 . Os motivos da não geocodificação de 433 casos (3\%) foram inexistência de endereço ou inadequação do endereço com a base cartográfica.

A análise de componentes principais com as variáveis socioeconômicas utilizadas produziu um fator socioeconômico responsável por $87 \%$ da variação total, que pode ser interpretado como um escore com a seguinte composição: 0,97 x (anos de instrução médios das pessoas responsáveis pelos domicílios) $+0,94 \mathrm{x}$ (anos de instrução médios das mulheres responsáveis pelos domicílios) $+0,85 \times$ (renda média das pessoas responsáveis pelos domicílios) $+0,85 \times$ (renda média das mulheres responsáveis pelos domicílios $)+(-) 0,89$ $\mathrm{x}$ (proporções de pessoas analfabetas) $+(-) 0,89 \mathrm{x}$ (proporção de mulheres analfabetas) + (-)0,56 x (proporção de domicílios com cinco ou mais moradores). A Tabela 1 apresenta a caracterização de cada um dos agrupamentos de setores censitários urbanos obtidos a partir do fator socioeconômico.

A Figura 1 mostra o mapa gerado pelo fator e as respectivas incidências para cada um dos níveis, em dois períodos distintos. Nota-se que no ano 1994-
1995, a componente socioeconômica foi relevante nas incidências de dengue em diversos setores, e o risco de adoecer nos locais de nível 4 foi aproximadamente 2,7 vezes maior do que naqueles de nível 1. A Figura 1 mostra ainda as incidências do quadriênio 1994-1998, que inclui o ano acima descrito. Neste primeiro quadriênio, a componente socioeconômica já não se mostrou um fator preponderante para as incidências de dengue nos diversos setores censitários do município.

Na Figura 2 apresenta-se o ano 2000-2001, quando houve a maior incidência da série histórica e o período final do estudo (1998-2002). No quadriênio correspondente a 1998-2002, as incidências não guardaram relação com a componente socioeconômica, uma vez que as maiores incidências ocorreram dentro dos níveis 2 e 1 , respectivamente, os agrupamentos de médio e melhor nível socioeconômico.

Na Tabela 2 estão dispostas as incidências de todos os períodos entre 1994 e 2002 e dos dois quadriênios, segundo a divisão do município por quartis. Verifica-se uma mesma tendência para todos os períodos (exceto 1994-1995), em que a transmissão de dengue ocorreu de forma independente em relação aos extratos socioeconômicos, chegando a atingir incidências maiores nos setores com classes de melhor nível, como pode ser observado nos anos 1997-1998, 1999-2000, 20002001 e 2001-2002.

\section{DISCUSSÃO}

O espaço social organizado tem grande importância no estudo das incidências e prevalências de doenças. Ao identificar áreas homogêneas, as ações coletivas para a prevenção de doenças podem ser enfatizadas, aumentando seu impacto nas comunidades afetadas. ${ }^{6,8}$

A construção de um indicador de risco urbano de transmissão de dengue é de grande importância no que tange estratégias de controle. Braga et $\mathrm{al}^{3}$ e Marzochi $^{11}$ ressaltam que existe a influência de vários elementos envolvidos no processo de transmissão e a necessidade de uma avaliação holística do fenômeno em áreas endêmicas, como São José do Rio Preto. ${ }^{13}$ Um desses elementos é o nível socioeconômico da população. Estudos apontam resultados discordantes, pois a questão da relação entre transmissão de dengue, medida pela incidência ou prevalência, e níveis socioeconômicos é controversa.

Pontes, ** para a cidade de Ribeirão Preto, SP, Medronho, ${ }^{12}$ para a cidade do Rio de Janeiro, RJ, Caiaffa et al, ${ }^{4}$ para a cidade de Belo Horizonte, MG e Siqueira et al, ${ }^{19}$ para a cidade de Goiânia, GO, mostraram haver

* Ministério da Saúde. DATASUS. Brasília; 2007: Informações de Saude - Demograficas e Socioeconomicas. [29/10/2007] Disponível em: http://tabnet.datasus.gov.br/cgi/deftohtm.exe?ibge/cnv/popsp.def

** Pontes RJS. Estudo da epidemia de dengue no município de Ribeirão Preto, SP, 1991-1992 [tese de doutorado]. Ribeirão Preto: Faculdade de Medicina de Ribeirão Preto da USP; 1992. 
Tabela 1. Características socioeconômicas e de saneamento básico dos quatro agrupamentos de setores censitários urbanos. São José do Rio Preto, SP, 2000.

\begin{tabular}{lcccc}
\hline \multirow{2}{*}{ Variável } & \multicolumn{3}{c}{ Agrupamento de setor censitário } \\
& I & II & III & IV \\
\hline Anos de instrução médio das pessoas responsáveis pelos domicílios & 10,8 & 7,9 & 6,5 & 5,3 \\
Anos de instrução médio das mulheres responsáveis pelos domicílios & 10,0 & 7,2 & 5,8 & 4,6 \\
Renda média (em reais*) das pessoas responsáveis pelos domicílios & $2.401,42$ & $1.046,99$ & 690,03 & 465,20 \\
Renda média (em reais*) das mulheres responsáveis pelos domicílios & $1.510,40$ & 687,20 & 453,60 & 298,50 \\
Proporção de pessoas responsáveis analfabetas (\%) & 3,2 & 5,8 & 8,1 & 11,3 \\
Proporção de mulheres responsáveis analfabetas (\%) & 3,6 & 6,6 & 9,2 & 12,4 \\
Proporção de domicílios com 5 ou mais moradores (\%) & 13,7 & 15,5 & 17,2 & 23,5 \\
Proporção de domicílios com água encanada (\%) & 100,0 & 99,8 & 99,9 & 99,9 \\
Proporção de domicílios com esgotamento sanitário (\%) & 99,9 & 99,8 & 99,3 & 99,2 \\
Proporção de domicílios com coleta regular de lixo (\%) & 99,9 & 99,9 & 99,3 & 99,7 \\
\hline
\end{tabular}

* Valores de setembro de 2000

Tabela 2. Coeficientes* de incidência de dengue, segundo períodose agrupamentos socioeconômicos. São José do Rio Preto, SP, 1994 a 2002.

\begin{tabular}{lcccc}
\hline Período & \multicolumn{4}{c}{ Agrupamento socioeconômico } \\
Ano/ Quadriênio & Nível 1 & Nível 2 & Nível 3 & Nível 4 \\
\hline $1994-1995$ & 245,8 & 349,8 & 398,8 & 658,8 \\
$1995-1996$ & 564,8 & 520,7 & 438,2 & 582,3 \\
$1996-1997$ & 61,5 & 37,0 & 73,8 & 72,6 \\
$1997-1998$ & 232,0 & 223,9 & 167,7 & 195,0 \\
$1998-1999$ & 761,5 & 864,0 & 678,3 & $1.001,6$ \\
$1999-2000$ & 160,4 & 183,4 & 126,1 & 66,6 \\
$2000-2001$ & $1.919,5$ & $2.210,1$ & $1.906,4$ & $1.762,5$ \\
$2001-2002$ & 282,2 & 332,1 & 280,7 & 218,7 \\
$1994-1998$ & $1.104,5$ & $1.125,3$ & $1.067,7$ & $1.485,7$ \\
$1998-2002$ & $3.169,1$ & $3.642,6$ & $3.038,1$ & $3.083,8$ \\
\hline *por 100.000 habitantes & & &
\end{tabular}

associação positiva entre maiores riscos de contrair dengue e piores níveis socioeconômicos da população. Costa \& Natal, ${ }^{7}$ avaliando a transmissão de dengue em São José do Rio Preto, SP, em 1995, identificaram essa mesma relação, resultados concordantes com os do presente estudo para o período de setembro de 1994 a agosto de 1995 .

Reiter et al, ${ }^{17}$ ao estudarem duas cidades vizinhas (Nueva Laredo no México e Laredo nos Estados Unidos), identificaram maior incidência de dengue na cidade mexicana apesar de o vetor Aedes aegypti ser mais abundante na cidade americana. Esses autores atribuíram esse paradoxo a fatores econômicos, como o maior uso de aparelhos de ar condicionado em Laredo. Oliveira \& Valla ${ }^{15}$ discutiram a relação entre mobilização popular e controle de dengue, apontando a relação entre a precariedade dos serviços de saneamento básico em favelas da cidade do Rio de Janeiro e a emergência de epidemias de dengue.

Entretanto outros estudos apresentaram resultados discordantes. Vasconcelos et $\mathrm{a}^{23}$ identificaram maior soroprevalência de dengue na população com maior renda e escolaridade em Fortaleza, Ceará; explicam tais achados em função do hábito das pessoas mais favorecidas em cultivar plantas aquáticas e uso de descartáveis. Resultados semelhantes foram encontrados por Vasconcelos et $\mathrm{al}^{22}$ em São Luís, Maranhão. Teixeira et $\mathrm{al}^{20}$ para a cidade de Salvador, BA, observaram altas taxas de soroincidência em todas as áreas e níveis socioeconômicos estudados, concluindo que o risco de contrair dengue era similar em todas as áreas da cidade. Bartley et al, ${ }^{2}$ estudando a soroprevalência de dengue e encefalite japonesa no sul do Vietnam, não encontraram associação destas com níveis educacionais ou com a posse de aparelho de televisão. Espinoza-Gomés et $\mathrm{al}^{10}{ }^{10}$ também não encontraram correlação entre infecção recente por dengue e nível socioeconômico na Cidade de Colima no México.

No caso de São José do Rio Preto, as variáveis socioeconômicas foram explicativas para as incidências de dengue no período que compreendeu 1994-1995, resultado coerente com o encontrado por Costa \& Natal. ${ }^{7}$ No entanto, isso não se repetiu nos anos posteriores do estudo.

Uma primeira questão a ser levantada é se os quatro agrupamentos de setores censitários representaram áreas socioeconômicas distintas. A análise da Tabela 1 mostra que as características socioeconômicas dos quatro agrupamentos são coerentes com a classificação adotada. A utilidade dessa divisão pode ser constatada no estudo realizado por Vendramini et $\mathrm{al}^{24}$ sobre incidência de tuberculose em São José do Rio Preto, doença reconhecidamente associada a piores níveis socioeconômicos. Esse estudo ${ }^{24}$ utilizou os mesmos agrupamentos de setores censitários do presente estudo, e mostrou que a área com o pior nível socioeconômico 


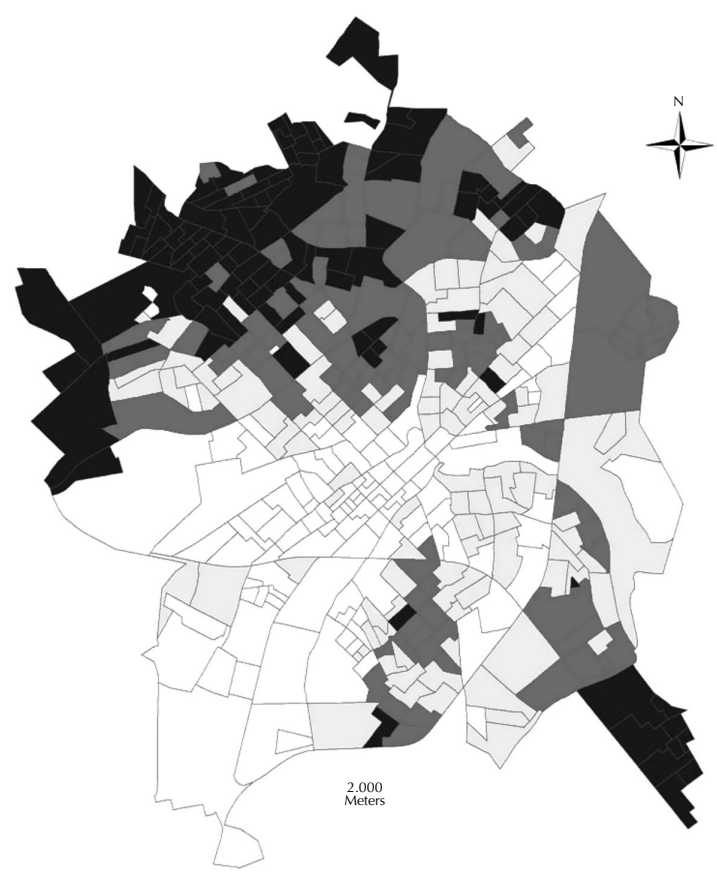

Dengue incidências 00/01*

$(*)$ por 100.000 habitantes

Nível socioeconômico 1: 1.919,5

Nível socioeconômico 2: 2.210,1

Nível socioeconômico 3: 1.906,4

Nível socioeconômico 4: 1.762,5

Dengue incidências 98/02*

$(*)$ por 100.000 habitantes

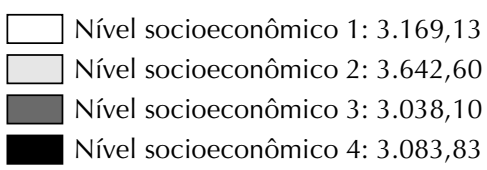

Figura 1. Mapa dos coeficientes de incidência de dengue para os agrupamentos de níveis socioeconômicos. São José do Rio Preto, SP, ano 1994/1995 e quadriênio 1994/1998.

apresentou a taxa mais elevada. Assim, o problema não está relacionado com a divisão adotada.

A própria Tabela 1 pode revelar o porquê do nível socioeconômico não ser um fator explicativo para a ocorrência de dengue em São José do Rio Preto. Apesar de diferenças entre as características socioeconômicas entre os quatro agrupamentos, do ponto de vista de variáveis representativas do saneamento básico, todas elas apresentam situações bastante favoráveis; pois as proporções de domicílios com água, esgoto e coleta regular de lixo são superiores a 99\%. Esses níveis de cobertura poderiam explicar tanto a similaridade nas incidências de dengue entre os setores de São José do Rio Preto quanto a emergência de epidemias de dengue em áreas de favelas no Rio de Janeiro. ${ }^{15}$ Mas, não explicam, por exemplo, a falta de relação entre níveis

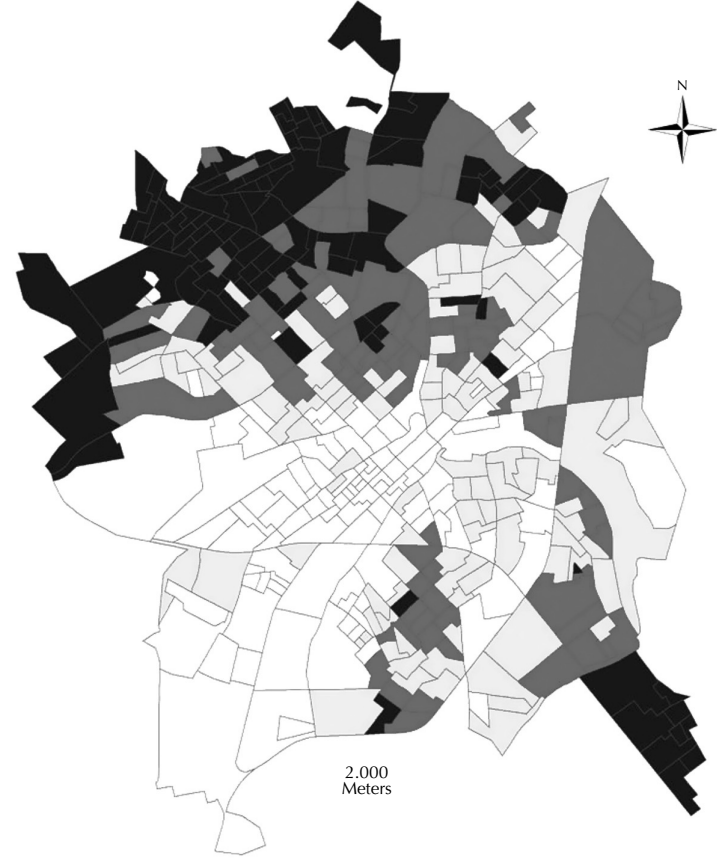

Dengue incidências 94/95*

$\left({ }^{*}\right)$ por 100.000 habitantes

Nível socioeconômico 1: 245,84

Nível socioeconômico 2: 349,84

Nível socioeconômico 3: 398,80

Nível socioeconômico 4: 658,81

Dengue incidências 94/98*

$\left(^{*}\right)$ por 100.000 habitantes

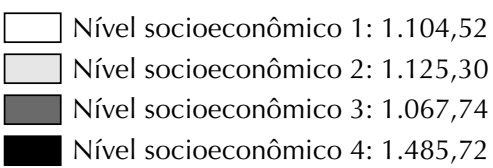

Figura 2. Mapa dos coeficientes de incidência de dengue para os agrupamentos de níveis socioeconômicos. São José do Rio Preto, SP, ano 2000/01 e quadriênio 1998/2002.

socioeconômicos e incidência de dengue em Salvador: o estudo realizado por Teixeira et $\mathrm{al}^{20}$ envolveu 30 áreas sentinelas, cinco delas tinham menos de $50 \%$ dos domicílios com as condições mínimas de saneamento. De toda maneira, esta é uma questão que precisa ser mais bem investigada.

Uma possível explicação para as discrepâncias entre os resultados dos estudos citados seria o fato de alguns terem se baseado nas informações das fichas de notificação compulsória dos sistemas de vigilância epidemiológica e outros em dados provenientes de estudos sorológicos. Contudo, parece não ser o caso, pois todos os estudos foram baseados em dados de notificação e em sorologia.

Uma discussão importante seria sobre a existência ou não de diferenças nos níveis de infestação do Aedes 
aegypti em função dos níveis socioeconômicos. Infelizmente poucos estudos nesta área têm sido realizados. Costa* investigou a relação entre transmissão de dengue, infestação larvária e níveis socioeconômicos em São José do Rio Preto, em 1995. Apesar de ter encontrado associação da incidência de dengue com níveis socioeconômicos da população, os níveis de infestação medidos pelo índice de Breteau não se mostraram diferentes entre as diferentes áreas socioeconômicas trabalhadas.

Estudo realizado em São José do Rio Preto, ${ }^{9}$ com dados de infestação medidos em janeiro de 2005 para a área urbana do município, também não identificou diferenças nos índices de Breteau em regiões de diferentes níveis socioeconômicos. Os resultados dos estudos realizados por Costa* e Ferreira \& Chiaravalloti-Neto ${ }^{9}$ são coerentes com a falta de relação encontrada entre dengue e níveis socioeconômicos.

Ainda em relação ao vetor, nos agrupamentos de setores mais favorecidos socioeconomicamente existem terrenos e áreas constituídas maiores, o que pode dificultar um cuidado mais minucioso por parte do morador. Apesar de as residências nos setores de nível 3 e 4 possuírem terrenos menores, há a ocorrência de maior número de recipientes potenciais para a proliferação do vetor, cuja grande parte não é eliminada por ainda ter alguma utilidade para os moradores, principalmente, venda ou doação. ${ }^{5}$

Uma questão a ser mais bem esclarecida é o porquê do encontro de diferenças entre os níveis socioeconômicos apenas no período de 1994-1995, quando ocorreu a primeira grande epidemia de dengue em São José do Rio Preto. Mondini et $\mathrm{al}^{13}$ constataram que no primeiro quadrimestre daquele período a transmissão iniciou-se em poucos setores na área norte do município e estendeu-se, posteriormente, pelo restante do município. Provavelmente as ações de controle que foram desencadeadas após o início da transmissão tiveram menor efetividade nessa região. Dessa forma, a constatação de que as maiores incidências de dengue fossem encontradas nos setores com menor nível socioeconômico era esperada para o período. Isso porque é na zona norte de São José do Rio Preto que se encontram os agrupamentos com a população mais carente do município e onde primordialmente houve a introdução do vírus.

De toda maneira, a relação entre níveis socioeconômicos e transmissão de dengue precisa ser mais bem investigada e, talvez, ela dependa da realidade de cada município. No caso de São José do Rio Preto, é possível que as diferentes incidências estariam relacionadas a outros fatores que não as condições socioeconômicas.

Para Barcellos \& Bastos, ${ }^{1}$ "a categoria espaço tem valor intrínseco na análise das relações entre saúde e ambiente e no seu controle; conhecer a estrutura e dinâmica espacial permite a caracterização da situação em que ocorrem eventos de saúde". No presente caso, trata-se dos diferentes coeficientes de incidências de dengue encontrados em São José do Rio Preto e em outras localidades. Assim, é importante que sejam verificadas as relações espaciais entre a transmissão de dengue e outras variáveis além do nível socioeconômico, como o grau de imunidade da população em relação à linhagem viral circulante; ${ }^{14}$ local de infecção (moradia, estudo, trabalho); efetividade das medidas de controle; grau de aglomeração populacional; níveis de infestação vetorial; grau de verticalização das construções urbanas; indicadores de saneamento ambiental, hábitos e atitudes da população, entre outros.

\section{AGRADECIMENTOS}

A Bruno Galli da Empresa Municipal de Processamento de Dados, pela orientação no uso do ArcGis 9.0 e colaboração na confecção dos mapas.

* Costa AIP. Identificação de unidades ambientais urbanas como condicionantes da ocorrência de Aedes aegypti (Diptera: Culicidae) e de dengue na cidade de São José do Rio Preto, SP, em 1995 [dissertação de mestrado]. São Paulo: Faculdade de Saúde Pública da USP; 1995. 


\section{REFERÊNCIAS}

1. Barcelos C, Bastos FI. Geoprocessamento, ambiente e saúde: uma união possível? Cad Saude Publica. 1996;12 (3):389-97.

2. Bartley LM, Carabin H, Vinh Chau N, Ho V, Luxemburger C, Hien TT, et al. Assessment of the factors associated with flavirus eroprevalence in a population in Southern Vietnam. Epidemiol Infect. 2002;128(2):213-20.

3. Braga C, Ximenes RAA, Albuquerque MFPM, Souza WV, Miranda J, Brayner F, et al. Avaliação de indicador sócio-ambiental utilizado no rastreamento de áreas de transmissão de filariose linfática em espaços urbanos. Cad Saude Publica. 2001;17(5):1211-8.

4. Caiaffa WT, Almeida MC, Oliveira CD, Friche AA, Matos SG, Dias MA, et al. The urban environment from the health perspective: the case of Belo Horizonte, Minas Gerais, Brazil. Cad Saude Publica. 2005;21(3):958-967.

5. Chiaravalloti-Neto F, Fiorin AM, Conservani DT, Cesarino MB, Barbosa AAC, Dibo MR, et al. Controle do vetor do dengue e participação da comunidade em Catanduva, São Paulo, Brasil. Cad Saude Publica. 2003;19(6):1739-49.

6. Chiesa AM, Westphal MF, Kashiwagi NM. Geoprocessamento e a promoção de saúde: desigualdades sociais e ambientais em São Paulo. Rev Saude Publica. 2002;36(5): 559-67.

7. Costa AIP, Natal D. Distribuição espacial da dengue e determinantes socioeconômicos em localidade urbana no sudeste do Brasil. Rev Saude Publica. 1998;32(3):232-6.

8. D'Orsi E, Carvalho MS, Cruz OG. Similarity between neonatal profile and socioeconomic index: a spatial approach. Cad Saude Publica. 2005;21(3):786-94.

9. Ferreira AC, Chiaravalloti-Neto F. Infestação de área urbana por Aedes aegypti e relação com níveis socioeconômicos. Rev Saude Publica 2007;41(6):91522.

10. Espinoza-Gómez F, Hernández-Suárez CM, RendónRamírez R, Carrillo-Alvarez ML, Flores-González JC. Transmisión interepidémica del dengue en la ciudad de Colima, México. Salud Publica Mex. 2003;45(5):365-70.

11. Marzochi KBF. Dengue endémico: o desafio das estratégias de vigilância. Rev Soc Bras Med Trop. 2004;37(5): 413-5

12. Medronho AR. Geoprocessamento e saúde: uma nova abordagem do espaço no processo saúde doença. Rio de Janeiro: Fundação Oswaldo Cruz; 1995.
13. Mondini A, Chiaravalloti-Neto F, Gallo Y Sanches M, Lopes JCC. Análise espacial da transmissão de dengue em cidade de porte médio do interior paulista. Rev Saude Publica. 2005;39(3): 444-51.

14. Newton EA, Reiter P. A model of the transmission of dengue fever with an evaluation of the impact of ultralow volume (ULV) insecticide applications and dengue epidemics. Am J Trop Med Hyg. 1992;47(6):709-20.

15. Oliveira RM, Valla VV. As condições e as experiências de vida de grupos populares no Rio de Janeiro: repensando a mobilização popular no controle do dengue. Cad Saude Publica. 2001;17(Supl):77-88.

16. Organização Mundial da Saúde. Dengue hemorrágica: diagnóstico, tratamento, prevenção e controle. $2^{\mathrm{a}} \mathrm{ed}$. São Paulo; 2001.

17. Reiter $P$, Lathrop S, Bunning M, Biggerstaff B, Singer $\mathrm{D}$, Tiwari T, et al. Texas lifestyle limits transmission of dengue virus. Emerg Infect Dis. [periódico na internet] 2003[ Acesso em 4/7/2006];9(1):86-9. Disponível em: http://www.cdc.gov/ncidod/eid/vol9no1/02-0220.htm

18. Rigau-Pérez JG, Clark GG, Gubler DJ, Reiter P, Sanders EJ, Vorndam AV. Dengue and dengue haemorrhagic fever. Lancet. 1998;352(9132):971-7.

19. Siqueira JB, Martelle CM, Maciel IJ, Oliveira RM, Ribeiro MG, Amorim FP, et al. Household survey of dengue infection in Central Brazil: spatial point pattern analysis and risk factors assessment. Am J Trop Med Hyg. 2004;71(5):646-51.

20. Teixeira MG, Barreto ML, Costa MCN, Ferreira LD, Vasconcelos PF, Cairncross S. Dynamics of dengue virus circulation: a silent epidemic in a complex urban area. Trop Med Int Health. 2002;7(9):757-62.

21. Tauil PL. Urbanização e ecologia do dengue. Cad Saude Publica. 2001; 17(Supl):99-102.

22. Vasconcelos PFC, Lima JWO, Raposo ML, Rodrigues SG, Rosa JFST, Amorin SMC, et al. Inquérito soroepidemiológico na Ilha de São Luis durante epidemia de dengue no Maranhão. Rev Soc Bras Med Trop. 1999;32(2):171-9.

23. Vasconcelos PFC, Lima JWO, Rosa APAT, Timbó MJ, Rosa EST, Lima HR, et al. Epidemia de dengue em Fortaleza, Ceará: inquérito soro-epidemiológico aleatório. Rev Saude Publica. 1998;32(5):447-54.

24. Vendramini SH, Santos ML, Gazetta CE, ChiaravallotiNeto F, Ruffino-Neto A, Villa TC. Tuberculosis risks and socio-economic level: a case study of a city in the Brazilian south-east, 1998-2004. Int J Tuberc Lung Dis. 2006;10(11):1231-5.

A Mondini foi apoiado pela Fundação de Amparo à Pesquisa do Estado de São Paulo (Fapesp - Processo no 03/12151-1; bolsa de mestrado). Baseado na dissertação de mestrado de A Mondini, apresentada à Faculdade de Medicina de São José do Rio Preto, em 2005. 